\title{
Assessing Changes in Cognitive Function and Quality of Life associated with Exercise in Individuals with Parkinson's Disease and their Caregivers
}

\author{
Lesley A Clack*, Jodi Ashman, Christine Widmer and Bryan L Riemann
}

Department of Health Sciences, Armstrong State University, USA

Received: August 14, 2017; Published: September 06, 2017

*Corresponding author: Lesley Clack, Department of Health Policy and Management, University of Georgia, 100 Foster Rd, Athens, GA 30602, USA, Tel: 229-322-7080; Email: lesleyaclack@gmail.com

\begin{abstract}
The purpose of this study was to examine the effects of a community-based, multi-modal exercise program on cognitive functioning and quality of life in individuals with Parkinson's disease and their caregivers. Assessments were administered pre and post participation in the program to assess changes in psychosocial functioning and changes in cognitive functioning in individuals with Parkinson's and their caregivers. Results for individuals with Parkinson's were statistically significant for both assessments while the change in scores from pre-test to post-test was not statistically significant for caregivers. The results of the study highlight the benefits of the program for individuals with Parkinson's.
\end{abstract}

Keywords: Parkinson's; Cognitive; Caregiver; Quality of Life; Exercise

\section{Introduction}

Parkinson's disease is a degenerative neurological disorder which currently lacks a cure. This disorder is usually characterized by both motor impairment, such as tremors, rigidity, and impaired balance and coordination, and non-motor impairment, such as cognitive impairment and reduced quality of life [1]. Approximately 1 out of every 100 people over the age of 60 is affected by Parkinson's disease [2], and 5 million people worldwide [3]. Not only do physical impairments occur during the early onset of Parkinson's disease, changes in cognitive function are also affected. While cognitive impairments related to Parkinson's disease primarily manifest as complications in daily executive functioning, the symptoms are often subtle and occur gradually. Symptoms such as depression have been documented as cognitive impairment increases and, due to the effect of the symptoms on daily life, these impairments can interfere with social and occupational lifestyles, often leading to job loss and a decrease in social interactions [4].

Although scientists and physicians continually debate on which is the best treatment for the symptoms of Parkinson's disease (deep brain stimulation, oral medication, etc.), a permanent solution to the disease has yet to be discovered. Physiotherapists and physicians have investigated the benefits of prescribing exercise programs to individuals with Parkinson's in an effort to slow the progression of Parkinson's disease symptoms [5]. While physical exercise has been shown to improve physical function [5], the effect on cognitive function and quality of life in individuals with Parkinson's disease has not been rigorously studied or well-documented. Most research studies available regarding Parkinson's disease and cognitive function focus on drug therapy as a solution [6]. Based on the costs and risks associated with drug therapy, studies seeking alternative solutions are needed.

There are a few studies that have reported the beneficial effects of exercise on cognition. Lilian Gobbi et al. [7] conducted a study to analyze the effects of various exercise programs on the psychological and cognitive functions in individuals with Parkinson's disease [7]. This study included 45 participants with Parkinson's disease, and thirty four of the participants completed $70 \%$ or more of the trial. The purposes of the programs were to promote psychosocial interaction through non-motor activities and to develop functional capacity through multi-modal exercise. The results showed that not only did continuous physical exercise for four months improve the cognitive and psychosocial functioning of the participants, but the individuals with Parkinson's disease in the control group had the same increase in cognitive functioning. This led the researchers to conclude that while physical exercise has been shown to increase physical and cognitive functioning in individuals with Parkinson's disease, social interaction promoted by group exercise can have a similar effect [7]. 
KE Cruise et al. [8] conducted a study involving 28 individuals with Parkinson's disease in an effort to determine if physical exercise had any effect on the cognitive functioning, mood, and diseasespecific quality of life factors for individuals with Parkinson's disease [8]. Fifteen were allocated to an exercise intervention program (EIP), while the remaining thirteen were designated as the control group. The EIP group conducted progressive anaerobic and aerobic exercise twice a week for 12 weeks, while the control group conducted their usual daily life. Results showed that exercise did have a positive influence on factors concerning the frontal lobe based executive functions of the brain, such as verbal fluency and spatial working memory. However, mood and disease-specific quality of life functions were shown to not improve by exercise [8].

Danielle Murray et al. conducted a systematic review of the literature to examine research findings regarding the effects of exercise on cognition in both animal and human participants with Parkinson's disease [5]. Their review included 14 reports / trials, six animal studies and eight human clinical studies. The researchers concluded that the clinical studies showed that several types of exercise (i.e. dance, aerobics, resistance training) improves cognitive function in individuals with Parkinson's disease. The researchers recommended that an exercise team should be recommended by providers as part of routine management and neuro-rehabilitation for individuals with Parkinson's disease [5]. These studies suggest a potential benefit of general exercise on cognition that is worth further investigation.

Approximately $80 \%$ of individuals with Parkinson's disease experience cognitive impairment, which is distressing for both the individual with Parkinson's disease and their caregivers, and is associated with poorer quality of life in both individuals with Parkinson's disease and caregivers [9]. Quality of life in caregivers is typically lower than the general population due to the burden of caring for an individual with a debilitating disease [10]. Prior studies have shown that patient quality of life impairment is strongly associated with high levels of caregiver strain and burden [11]. While many studies have assessed the burden on caregiver quality of life, the question remains whether a structured exercise program may improve quality of life in caregivers.

The researchers of this study partnered with a local weightlifting centre that created a community-based, group multimodal exercise program for individuals with Parkinson's disease and their caregivers. The program offers weekly classes in which the participants complete a variety of training exercises including whole body movements, agility (ladder drills), strength training and boxing. The exercise program was not an intervention of the study. The purpose of this investigation was to determine if individuals with Parkinson's disease and their caregivers that are enrolled in the program are experiencing positive changes in cognitive function and quality of life after six weeks of participation.

\section{Methods}

This study was a pre-test/post-test design. Participants were assessed prior to exercise, and then after six weeks of exercise. Institutional Review Board approval was obtained prior to any testing.

\section{Participants}

Participants registered in the community-based, multimodal exercise program, both those with Parkinson's disease and their caregivers, were invited to participate in the study. For the individuals with Parkinson's disease, the Hoehn-Yahr scales (H-Y) and the General Practitioner Assessment of Cognition (GPCOG) scales were used for inclusionary purposes. The H-Y scale describes the progression of Parkinson's disease symptoms. A score of 4 or 5 indicates that symptoms are severely debilitating. The GPCOG is a screening tool for cognitive impairment, with a score below 4 indicative of significant cognitive impairment. Individuals with Parkinson's disease who met the following criteria were eligible for study participation:

\section{i. An H-Y score of 1 to 3;}

\section{ii. A GPCOG score of 5 to 9 .}

Caregiver participation was voluntary for those individuals with Parkinson's disease that met inclusionary criteria for the study. Prior to any study procedures; all participants were given a full overview of study procedures and provided written informed consent.

\section{Assessments}

For those individuals with Parkinson's disease that met the inclusionary criteria for the study, researchers administered the Parkinson's disease Questionnaire (PDQ-39) and the Scales for Outcomes in Parkinson's Disease- Cognition (SCOPA-COG). The Parkinson's disease Questionnaire (PDQ-39) was used to assess quality of life in individuals with Parkinson's disease. The PDQ39 is comprised of 39 self-report items over 8 subscales. Subscale scores and summary indexes are calculated and reported, with a higher score representing a lower quality of life [1]. The Scales of Outcomes in Parkinson's Disease-Cognition (SCOPA-COG) was used to assess cognitive function in individuals with Parkinson's disease. The SCOPA-COG is comprised of various components including memory, attention, executive functions, and visuospatial functions. A lower score indicates greater cognitive impairment [12].

Caregivers of the individuals' with Parkinson's disease that were willing to participate in the study completed the Short Form 36(SF-36) to assess their changes in their quality of life. The Short Form 36 (SF-36) was used to assess quality of life in caregivers. The SF-36 allows assessment of the same 8 subscales as the PDQ39 [13]. These tests were completed in separate rooms in order to reduce bias in answering. After 6 weeks of exercise in the program, participants returned for post-testing and were administered the same assessments. Following exploratory verification of the pretest/post-test score differences being normally distributed, paired $t$ tests were conducted on the PDQ-39, SCOPA-COG, and SF-36 scores. All analyses were conducted with $\alpha=.05$.

\section{Results}

Forty two participants, 28 individuals with Parkinson's disease and 14 caregivers participated in the exclusionary testing for the study. Three individuals were excluded from the study based on their GPCOG and H-Y Scores. Of the 25 individuals with Parkinson's 
and 13 caregivers that completed the pre-test, 14 individuals with Parkinson's disease(age: $72.1 \pm 8.1, \mathrm{H}-\mathrm{Y}$ scale: $2.4 \pm .8$ ) and 9 caregivers(age: 74.1 \pm 6.0 ) completed the post-test. The Parkinson's disease participants demonstrated a statistically significant improvement $\left(\mathrm{P}=.017,95 \% \mathrm{CI}_{\text {diff }}: 9\right.$ to $\left.7.8, \mathrm{~d}=.52\right)$ in function and well-being based on the PDQ-39 (Figure 1). Overall, $79 \%$ of Parkinson's disease participants showed an improvement and, of those participants, 55\% showed an improvement of more than five points in their scores (Table 1). Additionally, the Parkinson's disease participants also reported a statistically significant improvement $\left(\mathrm{P}=.047,95 \% \mathrm{CI}_{\text {diff }}: .01\right.$ to $\left.2.2, \mathrm{~d}=.30\right)$ in cognitive functioning based on the SCOPA-COG (Figure 2). Fifty percent of Parkinson's disease participants showed improvement in their SCOPA-COG scores and, of those, $43 \%$ improved by at least two points (Table 1 ). In contrast, there was no statistically significant change $\left(\mathrm{P}=.902,95 \% \mathrm{CI}_{\text {diff: }}-1.2\right.$ to $2.9, \mathrm{~d}=.03$ ) in the quality of life reported by the caregivers based on the SF-36 scores (Table 2) (Figure 3).

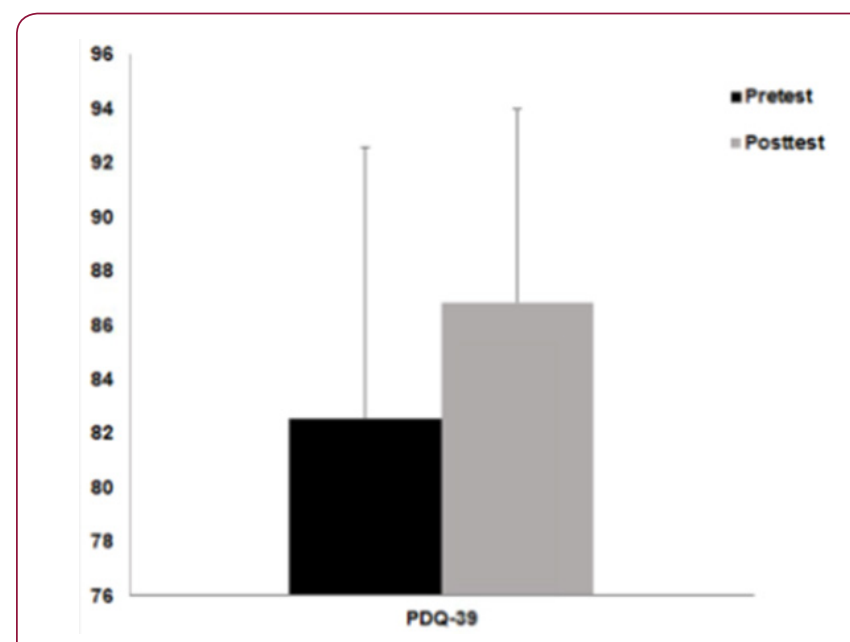

Figure 1: Pre-Test vs. Post-Test Scores for Parkinson's disease Participants on thePDQ-39.

Table 1: Parkinson's disease Participants' Assessment Scores.

\begin{tabular}{|c|c|c|c|c|}
\hline Participant & $\begin{array}{c}\text { Pretest } \\
\text { PDQ-39 }\end{array}$ & $\begin{array}{c}\text { Post PDQ- } \\
\mathbf{3 9}\end{array}$ & $\begin{array}{c}\text { Pretest } \\
\text { SCOPA-COG }\end{array}$ & $\begin{array}{c}\text { Post } \\
\text { SCOPA-COG }\end{array}$ \\
\hline P03 & 85.90 & 85.90 & 14 & 13 \\
\hline P05 & 88.46 & 95.51 & 9 & 8 \\
\hline P12 & 87.18 & 92.31 & 7 & 9 \\
\hline P17 & 90.38 & 86.54 & 7 & 7 \\
\hline P25 & 71.15 & 72.44 & 7 & 9 \\
\hline P26 & 81.41 & 87.82 & 14 & 18 \\
\hline P30 & 89.10 & 89.74 & 8 & 10 \\
\hline P32 & 87.82 & 92.31 & 10 & 10 \\
\hline P40 & 82.05 & 87.18 & 6 & 5 \\
\hline P44 & 82.05 & 92.31 & 10 & 11 \\
\hline P45 & 54.49 & 75.00 & 10 & 14 \\
\hline P51 & 87.82 & 91.03 & 13 & 15 \\
\hline P55 & 92.95 & 91.03 & 5 & 5 \\
\hline P61 & 74.36 & 76.92 & 11 & 11 \\
\hline
\end{tabular}

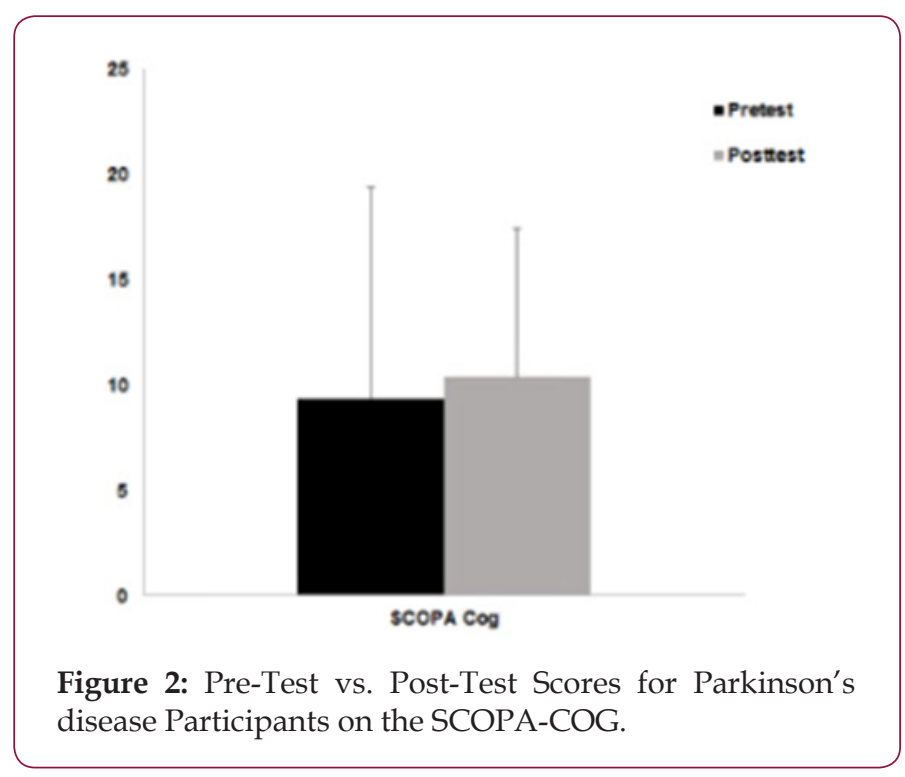

Table 2: Caregivers' Assessment Scores.

\begin{tabular}{|c|c|c|}
\hline Participant & Pre SF-36 & Post SF-36 \\
\hline C05 & 49.99 & 57.02 \\
\hline C12 & 56.78 & 55 \\
\hline C19 & 51.12 & 52.89 \\
\hline C20 & 44.59 & 45.35 \\
\hline C25 & 52.62 & 53.20 \\
\hline C32 & 56.96 & 57.00 \\
\hline C40 & 57.46 & 56.33 \\
\hline C45 & 50.31 & 50.82 \\
\hline C55 & 53.42 & 46.98 \\
\hline
\end{tabular}

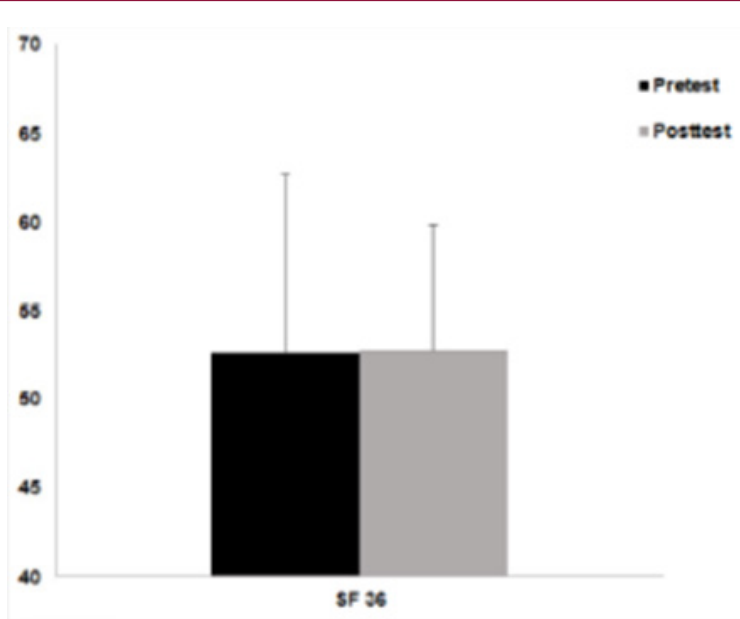

Figure 3: Pre-Test vs. Post-Test Scores for Caregivers on the SF-36. 


\section{Discussion}

This research considered the effects of a 6 week communitybased, multi-modal group exercise program on the cognitive functions and self-reported quality of life in individuals with Parkinson's disease. The main results of the research showed that at the end of six weeks of continuous exercise, cognitive functioning and quality of life scores increased for individuals with Parkinson's disease. Six weeks after beginning a dedicated exercise program, Parkinson's disease participants reported a significant increase in PDQ-39 scores, suggesting an overall improvement in quality of life. Further analysis of participant PDQ-39 scores showed that $55 \%$ of participants improved their scores by five points or more. This is a clinically meaningful increase that is consistent with other studies that have shown that motor and non-motor symptoms have a close relationship, and as one improves the other typically improves [14]. The results support the need for further research on the effects of increased physical functioning and the possibility of permanently incorporating a dedicated exercise routine into the treatment plan of individuals with PD. Additionally, based on results of the pretest and post-test SCOPA-COG scores, the exercise program also prompted increased cognitive functioning. When considering caregiver participants, there was not a change in self-reported quality of life as measured by the SF-36. Future research should consider if a more intense or longer exercise program can prompt greater improvement in caregiver quality of life.

Despite the positive results revealed, there were several limitations to research throughout the study. The exercise program was not actively controlled as part of the research. Thus, aspects of the exercise program such as modes, intensity, and compliance were not actively monitored or factored into the statistical analysis. For example, several participants with Parkinson's commented during post-testing that they had missed several of the exercise sessions. Despite missing several of the sessions, statistically significant improvements were still identified. Another limitation of the study was because it was not externally supported; participation incentives to attend the pre and post-testing were not available. Participants were responsible for their own transportation to the pre and post-testing site, and therefore affected the response rate. Another factor was that the majority of the participants and their caregivers are of retirement age, and was involved with a lot of travel. Thus, conflicts in schedules precluded some participants from returning for the post-test assessments. Regardless of the amount of limitations represented throughout the study, the data garnered from the participants still showed that at the end of six weeks of continuous exercise, cognitive functioning and quality of life scores increased in individuals with PD, which is notable.

\section{Conclusion}

The results of the research study showed that both cognitive functioning and quality of life measures increased in individuals with Parkinson's disease in response to a dedicated multi-modal exercise program. A recommendation for future research would be to conduct a study over a longer period of time in order to identify further increases in cognitive functioning and quality of life in correlation with longer exercise periods. Furthermore, future research studies could be conducted including the use of control groups in order to determine if group activities not revolving around exercise have a similar effect on cognitive functions and quality of life. Another recommendation for further study would be a comparison study analysing any differences between a group exercise setting versus an individual with Parkinson's disease conducting exercise alone at his / her own time and pace. These additions could determine the effects of a group atmosphere on cognitive functioning and quality of life versus solo exercise.

\section{References}

1. Martinez-Martin P, Jeukens-Visser M, Lyons KE, Rodriquez-Blazquez C, Selai C, et al. (2011) Health-Related Quality of Life Scales in Parkinson's Disease: Critique \& Recommendations. Movement Disorders 26(13): 2371-2380.

2. Yang Y, Tang B, Guo J (2016) Parkinson's Disease and Cognitive Impairment. Parkinson's Disease 1: 1-8.

3. Holden SK, Jones WE, Baker KA, Boersma IM, Kluger BM (2017) Outcome Measures for Parkinson's Disease Dementia: A Systematic Review. Movement Disorders- Clinical Practice 3(1): 9-18.

4. Frisina PG, Borod JG, Foldi NS, Tenenbaum HR (2008) Depression in Parkinson's Disease: Health Risks, Etiology, and Treatment Options. Neuropsychiatric Disease \& Treatment 4(1): 81-91.

5. Murray DK, Sacheli MA, Eng JJ, Stoessl AJ (2014) The Effects of Exercise on Cognition in Parkinson's Disease: A Systematic Review. Translational Neurodegeneration 3(1): 1-5.

6. Adamski N, Adler M, Opuis K, Penner I (2016) A Pilot Study on the Benefit of Cognitive Rehabilitation in Parkinson's Disease. Therapeutic Advances in Neurological Disorders 9(3): 153-164.

7. Gobbi LTB, Teixeira-Arroyo C, Lirani-Silva E, Vitorio R, Barbieri FA, et al. (2013) Effect of Different Exercise Programs on the Psychological and Cognitive Functions of People with Parkinson's Disease. Motriz, 19(3): 597-604.

8. Cruise KE, Bucks RS, Loftus AM, Newton RU, Pegoraro R, et al. (2011) Exercise and Parkinson's Benefits for Cognition and Quality of Life. Acta Neurologica Scandinavica 123(1): 13-19.

9. Lawson RA, Yamall AJ, Duncan GW, Breen DP, Khoo TK, et al. (2016) Cognitive Decline and Quality of Life in Incident Parkinson's Disease: The Role of Attention. Parkinsonism\& Related Disorders 27: 47-53.

10. Peters M (2014) Quality of Life and Burden in Caregivers for Patients with PD. Focus on Parkinson's Disease 24(1): 43-48.

11. Oguh O, Kwasny M, Carter J, Stell B, Simuni T (2013) Caregiver Strain in Parkinson's Disease: National Parkinson Foundation Quality Initiative Strategy. Parkinsonism \& Related Disorders 19(11): 975-979.

12. Das D, Biswas A, Roy A, Sauerbier A, Bhattacharyya KB (2016) Cognitive Impairment in Idiopathic Parkinson's Disease. Neurology India 64(3): 419-427.

13. Opara JA, Brola W, Leonardi M, Blaszcyk B (2012) Quality of Life in Parkinson's Disease. Journal of Medicine and Life 5(4): 375-381.

14. Lee HM, Koh SB (2015) Many Faces of Parkinson's Disease: Non-Motor Symptoms of Parkinson's Disease. Journal of Movement Disorders 8(2): 92-97. 


\begin{tabular}{ll}
\hline BIOMEDICAL & Assets of Publishing with us \\
RESEARCHES & Global archiving of articles \\
\hline & - Immediate, unrestricted online access \\
\hline
\end{tabular}

